\title{
Professional Domiciliary Oral Care for Elderly in Nursing Homes-A Randomized Controlled Pilot Trial
}

\author{
Petteri Sjögren1, Caroline Croonquist Girestam ${ }^{2,3}$, Pia Skott ${ }^{2,3}$, Nasita Marsson², \\ Renata Nova4, Mikael Zimmerman ${ }^{1,5}$, Inger Wårdh ${ }^{3,5 *}$ \\ ${ }^{1}$ Oral Care AB, Stockholm, Sweden \\ ${ }^{2}$ Folktandvården Stockholm AB, Stockholm, Sweden \\ ${ }^{3}$ Academic Centre of Geriatric Dentistry, Stockholm, Sweden \\ ${ }^{4}$ Nova Smile Dental AB, Stockholm, Sweden \\ ${ }^{5}$ Department of Dental Medicine, Karolinska Institutet, Huddinge, Sweden \\ Email: *inger.wardh@ki.se
}

Received 6 June 2016; accepted 22 August 2016; published 25 August 2016

Copyright (C) 2016 by authors and Scientific Research Publishing Inc.

This work is licensed under the Creative Commons Attribution International License (CC BY). http://creativecommons.org/licenses/by/4.0/

c) (†) Open Access

\begin{abstract}
Domiciliary dental care (DDC) makes regular dental visits possible for people with different functional limitations who otherwise would not be able to access a dental clinic. DDC also facilitates cooperation with nursing staff. To our knowledge, the effect of DDC on oral health among elderly people in nursing homes has not been studied. As part of a project to establish recommendations for DDC, the aim of this randomized, controlled, single-blind, pilot trial with three parallel arms was to study the effect of professionally conducted oral care intervention, in domiciliary setting, on gingival bleeding on probing, dental plaque and oral mucosa. For three months, the participants were given either A) monthly professional DDC with oral care intervention, B) monthly individual oral care instructions, or C) oral care as usual. A total of $102(n=107)$ individuals completed the study. After three months, gingival bleeding on probing was significantly reduced among more participants in groups $A$ and $B$ compared with group $C(p<0.0004)$. Dental plaque scores changed significantly over three months for group B compared with group $C(p<0.04)$. Mucosal-plaque scores were significantly reduced in group A compared with both group $B$ and $C$ ( $p<$ 0.0001). All other intergroup differences were statistically non-significant. Compared with care as usual, professional DDC has the most favourable effect on gingival bleeding, whereas individual oral care instructions result in the highest reduction of dental plaque. Both professional oral care interventions and individual oral-hygiene instructions should be included in a domiciliary oralcare programme.
\end{abstract}

Corresponding author.

How to cite this paper: Sjögren, P., Girestam, C.C., Skott, P., Marsson, N., Nova, R., Zimmerman, M. and Wårdh, I. (2016) Professional Domiciliary Oral Care for Elderly in Nursing Homes-A Randomized Controlled Pilot Trial. Health, 8, $1112-1119$. http://dx.doi.org/10.4236/health.2016.811116 


\section{Keywords}

\section{Geriatrics, Aged, Domiciliary Care, Oral Health, Oral Hygiene}

\section{Introduction}

An increasing number of research publications point to the association between oral and general health, and even mortality [1]-[3]. It is important to develop oral care strategies that effectively prevent oral diseases, especially in frail risk groups, such as very old and care dependent individuals, and it is also important to help these vulnerable groups gain access to professional oral health care.

Professional oral care has been considered an effective method to prevent dental diseases, such as caries and periodontal problems in elderly people. The national guidelines for dentistry in Sweden state that there is no scientific evidence for the effect of professional oral care in addition to daily self-performed oral care tasks in adults [4]. This statement, however, cannot be transferred to frail, elderly people who are dependent on help from their caregivers to perform their daily oral hygiene tasks. In addition, nursing staff in general have been found to have poor knowledge in oral care and lack of motivation to perform the oral care tasks [5] [6], with the risk of daily oral care tasks being handled by elderly individuals themselves [7].

Domiciliary dental care (DDC) makes regular dental visits possible for people with different functional limitations, who otherwise would not be able to transport themselves to a dental clinic. It is also a way to establish cooperation with overburdened nursing staff [8] who express extended dental contacts as a way to improve the oral care for their care takers [5] [9].

To our knowledge, the effect of DDC for elderly people in nursing homes has not been studied previously. High-quality DDC including preventive oral care can be performed with rather simple equipment, while more advanced DDC requires more advanced equipment [10]. However, the Swedish dental system's subsidies for dental care for elderly and/or care-dependent individuals have been increasingly restricted year by year in terms of the number of preventive, oral care procedures covered [11]. The levels of restrictions vary between different county councils and regions, and the rationale is probably economic and political.

\section{Aim}

As a part of a project to establish relevant recommendations for DDC, the aim of this randomized, controlled, single-blind pilot trial with three parallel arms, over three months, was to study the effect of domiciliary delivered oral care intervention on gingival bleeding on probing, dental plaque and oral mucosa. The participants were given either A) monthly DDC with professional oral care intervention, B) monthly individual oral care instructions, or C) oral care as usual.

\section{Methods}

\subsection{Participants and Eligibility}

During 2015, 125 individuals were assessed for eligibility to participate in the study. Participants were required to live in a nursing home, age $\geq 65$ years, to have at least ten natural teeth and/or dental implants, and to be able to cooperate during the interventions and registrations. Exclusion criteria were: edentulism, two complete dentures, cognitive impairment that would prevent cooperation, extreme hyposalivation (assessed by friction test with the back of a dental mirror) [12], malignancy, coagulation defect or haematological disorder.

\subsection{Ethics}

The study was approved by the Regional Ethical Review Board (Stockholm, Sweden 2014/672-31/4), and all participants provided written informed consent to participate in the study. The study design and criteria were not changed after the trial was initiated. The primary investigator (author IW) monitored for adverse events reported during the trial. 


\subsection{Study Design}

A randomized, controlled, single-blind trial with a total of 107 individuals in three parallel groups, see Figure 1 . The randomization was done by an independent study coordinator and the code was concealed from the study personnel who provided the interventions and analysed the data. Randomisation was done at nursing home level, with a 1:1:1 allocation ratio, including six different nursing homes in Stockholm, Sweden.

Two weeks before the baseline registration all chlorhexidine gluconate products were discontinued. At baseline registration, all participants received professional dental cleaning and debridement. The nursing home personnel were given a 20-to-30-minute lecture in oral hygiene. On a monthly basis during three months, the study participants were visited in their rooms at the nursing homes and received different oral care interventions as follows: Participants in group Areceived monthly professional dental cleaning, cleaning with interdental brushes and necessary supra-gingival scaling as well as individual oral care instructions, group B received monthly individual verbal and written oral care instructions (but no cleaning), while group C participants were not visited at all by dental personnel during the study period and continued with their personal oral care as usual. In all three study groups, the participants were given both verbal and written recommendations to brush their teeth with 1000 - 1450 ppm fluoride tooth paste and to do interdental cleaning daily, as usual. After three months, a final registration was made, and the study ended.

\subsection{Outcomes}

The primary outcome was bleeding on probing (BoP) according to Modified Sulcus Bleeding Index [13], in the buccal gingival crevice on Ramfjord's index teeth $(16,12,24,36,32,44)$ or, when missing, the closest tooth [14]. Secondary outcomes were the plaque index score and mucosal score, combined to a mucosal-plaque score

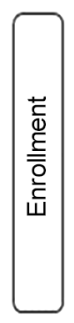

Excluded $(\mathrm{n}=18)$

- Not meeting inclusion criteria $(n=10)$

- Declined to participate $(n=8)$

- Other reasons $(n=0)$

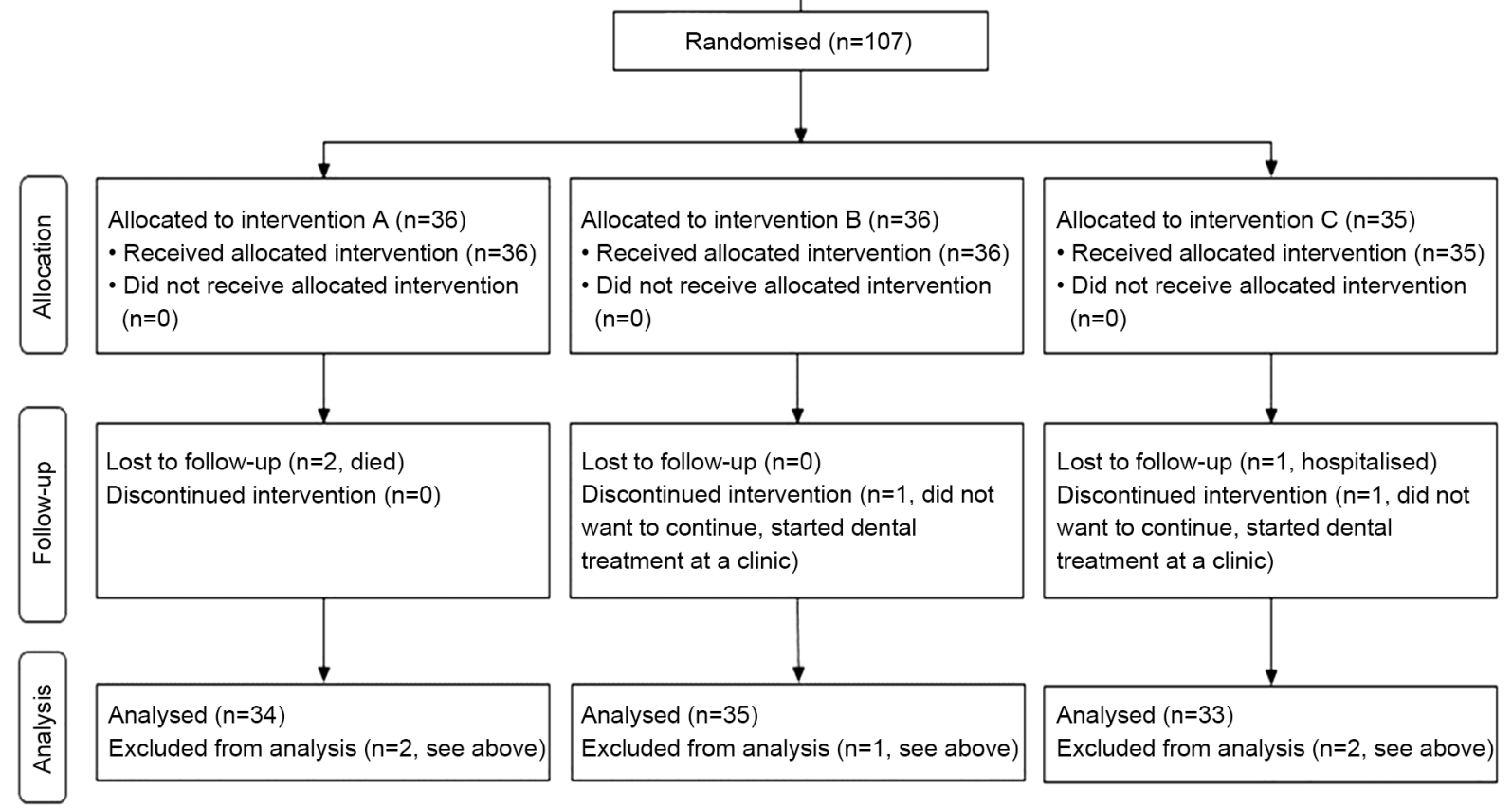

Figure 1. Consort diagram of the participant flow during the trial. 
(MPS) [15]. Xerostomia at baseline was assessed by asking the participant about subjective impressions of mouth dryness and by mirror-sliding friction test inside of the buccal mucosa [12]. Recordings were also made for oral care products used during the study, new oral care products bought during the study, use of salivastimulating or saliva-substituting substances, as well as the number of prescribed drugs at study start.

Before start, all three data collecting dental personnel were calibrated. Bleeding on probing was registered from 0 - 3, with 0 no bleeding, 1 slight bleeding on probing on certain crevices, 2 slight, general bleeding on probing, 3 heavy, visible bleeding on probing.

The MPS [15] was used both for individuals with and without natural teeth and prostheses. The MPS index was originally designed to evaluate oral health and oral hygiene in groups of elderly individuals in hospitals and at other institutions [15], and comprises two measurements; first, mucosal disorders (MS), using the criteria 1) normal gingiva and mucosa 2) mild inflammation 3) moderate inflammation and 4) severe inflammation; and second plaque score (PS), using the criteria 1) no easily visible plaque 2) small amounts of plaque 3) moderate amounts of plaque and 4) abundant amounts of confluent plaque. The scores are then added to a single index measuring a person's oral hygiene. The purpose of the scores is not to make a diagnosis [15].

\subsection{Statistical Analyses}

Baseline characteristics were presented as mean numbers with standard deviations (SD), or as proportions, and compared between study groups with Fischer's exact test. The limit for statistical significance was set to p < 0.05 .

The clinical outcomes were analysed as improvement in health status (positive values), no change (zero), or deterioration (negative values), in frequency tables, and as mean changes from baseline to three months' follow-up.

For the subsequently planned full-scale trial, a sample size of 175 individuals per group was calculated in order to detect $7 \%$ difference in $\operatorname{BoP}(\alpha=0.05$ och $\beta=0.20)$; thus this pilot study was intentionally underpowered.

\section{Results}

\subsection{Withdrawals and Dropouts}

The participant flow through the study is shown in Figure 1. In group A, two participants died during the study period. In groups B and C, one participant in each group discontinued treatment since they became motivated to start treatment at a dental clinic; and in group C, one participant was lost to follow-up due to hospitalisation, leaving a total of 102 participants for final analyses at three months' follow-up.

\subsection{Baseline Characteristics}

Baseline characteristics are shown in Table 1. The mean age of the participants was 86.8 (SD 7.8) years, with 61\% women. The mean number of natural teeth was 20.9 (SD5.2), and 23\% received help with their daily tooth brushing. During the last year, 79\% had regular dental appointments and $2 \%$ had an emergency dental visit. The participants had a mean number of 9.2 (SD 6.0) prescribed drugs and $28 \%$ had xerostomia.

\subsection{Bleeding on Probing}

At three months, the highest bleeding-on-probing category (score 3) was significantly improved (i.e. reduced) among more participants in groups A and B compared with group C ( $<<0.0004)$, whereas no significant differences were found between the groups regarding the lower bleeding-on-probing categories (scores 0 - 2) (Table 2).

\subsection{Plaque Score}

Measured by "improvement”, "no change” or "deterioration” for the individual plaque scores among the participants, the amount of biofilm changed significantly over three months in favour for group B (improved $\mathrm{n}=11$ ) compared with group $C$ (improved $n=7, p=0.0399)$ (Table 3 ). The mean plaque scores were reduced in groups A ( -0.25 , SD 0.50) and B (-0.28, SD 0.51), but not in group C (0.03, SD 0.66, p = 0.0493 A vs. C, and p $=0.0327$ B vs. C) (Table 4). 
Table 1. Baseline characteristics.

\begin{tabular}{|c|c|c|c|c|}
\hline & Group A $(n=36)$ & Group B $(n=36)$ & Group C $(n=35)$ & Total $(\mathrm{n}=107)$ \\
\hline Age, mean (SD) & $89.8(7.2)^{1}$ & $87.6(6.6)^{2}$ & $82.9(8.1)^{1,2}$ & $86.8(7.8)$ \\
\hline Men, number (\%) & $11(31)$ & $13(36)$ & $18(51)$ & $42(39)$ \\
\hline Natural teeth, mean (SD) & $20.9(4.8)$ & $21.8(3.6)$ & $20.0(6.8)$ & $20.9(5.2)$ \\
\hline Help with tooth brushing, number (\%) & $9(25)$ & $7(19)$ & $9(26)$ & $25(23)$ \\
\hline Uses fluoride toothpaste, number (\%) & $36(100)$ & $36(100)$ & $34(97)$ & 106 (99) \\
\hline Uses other oral care products, number (\%) & $16(44)$ & $16(44)$ & $13(37)$ & $45(42)$ \\
\hline Tooth brushing frequency per day, mean (SD) & $2.0(0.7)$ & $2.1(0.6)$ & $1.9(0.7)$ & $2.0(0.7)$ \\
\hline Removable denture on implants, number (\%) & $0(0)$ & $2(5.6)$ & $2(5.7)$ & $4(3.7)$ \\
\hline Removable partial denture, number (\%) & $1(2.8)$ & $1(2.8)$ & $1(2.9)$ & $3(2.8)$ \\
\hline Removable complete denture, number (\%) & $3(8.3)$ & $1(2.8)$ & $1(2.9)$ & $5(4.7)$ \\
\hline Regular dental appointments, number (\%) & $25(6.9)$ & $31(8.6)$ & $28(0.8)$ & $84(79)$ \\
\hline Emergency dental appointments, number (\%) & $1(2.8)$ & $0(0)$ & $1(2.9)$ & $2(1.9)$ \\
\hline Number of drugs, mean (SD) & $9.3(5.1)$ & $9.5(6.8)$ & $8.8(6.0)$ & $9.2(6.0)$ \\
\hline Xerostomia ${ }^{3}$, number (\%) & $10(28)$ & $9(25)$ & $11(31)$ & $30(28)$ \\
\hline Strained food, number (\%) & $2(5.6)$ & $1(2.8)$ & $1(2.9)$ & $14(13)$ \\
\hline Nutritional drinks intake, number (\%) & $3(8.3)$ & $4(11)$ & $7(20)$ & $14(13)$ \\
\hline
\end{tabular}

${ }^{1}$ Statistically significant difference between group A and C ( $\left.p=0.0003\right) ;{ }^{2}$ Statistically significant difference between group B and C ( $\left.p=0.009\right)$. All other intergroup differences were n.s; ${ }^{3}$ Assessed by asking the participant and by mirror sliding friction test inside of the buccal mucosa.

Table 2. Changes in bleeding-on-probing scores over three months (positive values indicate clinical improvement whereas negative values indicate deterioration).

\begin{tabular}{lcccc}
\hline & \multicolumn{3}{c}{ Mean (SD) } \\
\cline { 2 - 4 } & Group A ( $=34)$ & Group B ( $=35)$ & Group C ( $\mathrm{n}=33)$ & p-value \\
\hline BoP score 0 & $-0.38(1.54)$ & $0.34(2.31)$ & $-0.12(2.43)$ & 0.2453 \\
BoP score 1 & $-0.09(1.44)$ & $-0.49(2.57)$ & $-0.42(2.02)$ & 0.9980 \\
BoP score 2 & $0.35(0.81)$ & $0.74(3.35)$ & $0.39(2.40)$ & 0.3297 \\
BoP score 3 & $0.12(0.41)$ & $0.00(0.00)$ & $-0.18(0.46)$ & $<0.0004^{1}$
\end{tabular}

${ }^{1} \mathrm{p}$-value for difference between group A versus $\mathrm{C}$, and B versus $\mathrm{C}$.

\subsection{Mucosal Score}

There were no significant differences in mucosal health over three months, as measured by "improvement", "unchanged", or "deterioration" for individual mucosal scores among the participants (Table 3). The mean mucosal scores were numerically, but not significantly, reduced in group A (-0.03, SD 0.51), but not in group B (0.06, SD 0.41, n.s.) or group C (0.11, SD 0.47, n.s) (Table 4).

\subsection{Mucosal-Plaque Score}

Measured as the number of individuals with “improved”, “unchanged”, or “deteriorated” mucosal-plaque scores, the amount of biofilm and mucosal health combined changed significantly in favour of group A (improved $\mathrm{n}=$ 16) compared with group $B$ (improved $n=1, p<0.0001)$, and with group $C$ (improved $n=2)(p<0.0001)$ (Table 3). The mean mucosal-plaque scores were reduced in group A (-0.75, SD 1.13) but not in group B (0.56, SD 0.97, $\mathrm{p}=0.0001 \mathrm{~A}$ vs B), or in group C (1.00, SD 1.46, $\mathrm{p}=0.0001 \mathrm{~A}$ vs C) (Table 4). 
Table 3. Frequency table for change over three months in plaque score, mucosal score, and mucosal-plaque score (MPS) among the study participants.

\begin{tabular}{|c|c|c|c|c|}
\hline & \multicolumn{4}{|c|}{ Change over 3 months, n (\%) } \\
\hline & Group A $(n=34)$ & Group B $(n=35)$ & Group C $(n=33)$ & Total $(\mathrm{n}=102)$ \\
\hline \multicolumn{5}{|l|}{ Plaque score $^{1}$} \\
\hline Deterioration & $1(2.94)$ & $1(2.86)$ & $8(24.24)$ & 10 \\
\hline Unchanged & $23(67.65)$ & $23(65.71)$ & $18(54.55)$ & 64 \\
\hline Improvement & $10(29.41)$ & $11(31.43)$ & $7(21.21)$ & 28 \\
\hline \multicolumn{5}{|l|}{ Mucosal score } \\
\hline Deterioration & $4(11.76)$ & $4(11.43)$ & $6(18.18)$ & 14 \\
\hline Unchanged & $25(73.53)$ & $29(82.86)$ & $25(75.76)$ & 79 \\
\hline Improvement & $5(14.71)$ & $2(5.71)$ & $2(6.06)$ & 9 \\
\hline \multicolumn{5}{|l|}{ MPS $^{2}$} \\
\hline Deterioration & $1(2.94)$ & 12 (34.29) & $16(48.48)$ & 29 \\
\hline Unchanged & $17(50.00)$ & $22(62.86)$ & $15(45.45)$ & 54 \\
\hline Improvement & $16(47.06)$ & $1(2.86)$ & $2(6.06)$ & 19 \\
\hline
\end{tabular}

${ }^{1} \mathrm{p}=0.0399$ for difference between group B versus $\mathrm{C} ;{ }^{2} \mathrm{p}<0.0001$ for difference between group A versus B, and A versus C.

Table 4. Plaque scores, mucosal scores and mucosal-plaque scores at baseline, after three months, and change over 3 months.

\begin{tabular}{|c|c|c|c|}
\hline & \multicolumn{3}{|c|}{ Mean (SD) } \\
\hline & Group A $(n=34)$ & Group B $(\mathrm{n}=35)$ & Group C $(\mathrm{n}=33)$ \\
\hline \multicolumn{4}{|l|}{ Plaque scores } \\
\hline Baseline & $2.00(0.83)$ & $2.11(0.62)$ & $2.49(0.70)$ \\
\hline At 3 months & $1.75(0.77)$ & $1.83(0.70)$ & $2.51(0.61)$ \\
\hline Change & $-0.25(0.50)^{1}$ & $-0.28(0.51)^{2}$ & $0.03(0.66)^{1}$ \\
\hline \multicolumn{4}{|c|}{ Mucosal scores } \\
\hline Baseline & $1.61(0.77)$ & $1.86(0.76)$ & $2.06(0.76)$ \\
\hline At 3 months & $1.58(0.65)$ & $1.92(0.77)$ & $2.17(0.75)$ \\
\hline Change & $-0.03(0.51)$ & $0.06(0.41)$ & $0.11(0.47)$ \\
\hline \multicolumn{4}{|c|}{ Mucosal-plaque scores } \\
\hline Baseline & $1.94(1.17)$ & $1.28(0.51)$ & $1.57(0.61)$ \\
\hline At 3 months & $1.19(0.40)$ & $1.83(1.00)$ & $2.57(1.54)$ \\
\hline Change & $-0.75(1.13)^{3,4}$ & $0.56(0.97)^{3}$ & $1.00(1.46)^{4}$ \\
\hline
\end{tabular}

Plaque scores range from 1 to 4 , where higher scores indicate more biofilm. Mucosal scores range from 1 to 4 , where higher scores indicate more mucosal inflammation. Mucosal-Plaque Score is the sum of plaque- and mucosal scores ranging from 2 to $8 ;{ }^{1} \mathrm{p}=0.0493$ for difference between group $\mathrm{A}$ and $\mathrm{C} ;{ }^{2} \mathrm{p}=0.0327$ for difference between group $\mathrm{B}$ and $\mathrm{C} ;{ }^{3} \mathrm{p}=0.0001$ for difference between group $\mathrm{A}$ and $\mathrm{B} ;{ }^{4} \mathrm{p}<0.0001$ for difference between group $\mathrm{A}$ and $\mathrm{C}$.

\subsection{Adverse Events}

No adverse events were reported during the trial.

\section{Discussion}

This study was the first in a comprehensive project with the aim to establish relevant recommendations for do- 
miciliary, preventive, professional oral care. In this randomized, controlled pilot trial, the participants were given either monthly professional domiciliary oral care, or monthly individual oral care instructions, compared with oral care as usual.

At three months' follow up, bleeding on probing on the highest level was significantly improved among more participants in both the professional, domiciliary oral care group, and in the monthly, individual oral care instructions group, compared with the care-as-usual group. In addition, dental plaque scores changed significantly over three months in favour of the instruction group compared with the control group, whereas mucosal-plaque scores were significantly reduced in the oral care group compared with both the instruction group and the care-as-usual group. A possible explanation for this result is that many elderly individuals do not immediately respond to dental plaque with development of gingivitis, which may reflect a decreased immune response [16]. However, there have been reports on the association between self-reported bleeding gums and cardiovascular diseases, while such a result may have implications for the general health [17].

Oral care instructions do not reach all nursing home staff at the same time. Staff attendance to instruction sessions varies with working schedules, but also because the oral care instructions are considered mandatory. Thus, during the study the least motivated staff, who would probably have gained most of the instructions, did not attend the instruction sessions. Further on, the need of regularly given oral care information seems to be relatively constant over time, given the high staff turnover and work load at nursing homes in large cities in Sweden. Furthermore, oral care tasks are nowadays difficult due to many natural teeth, and studies report that it seems easier to clean dentures [18]. Maybe oral care of today is so demanding that it is almost an impossible task for non-dental professionals alone to perform oral care properly for those who need help.

Another contributing reason may be that many of the care-dependent elderly themselves do not consider it natural that oral care tasks are assisted by nursing staff. They recognise oral care as a responsibility for dental professionals. Several research reports have dealt with the problem of non-cooperating nursing-home residents in the oral care situation $(5,7)$.

Generally,oral care protocols are designed like care bundles with a variety of interventions and activities, making it difficult to distinguish which part of the effect is derived from each of the given interventions, or whether the observed effect is a result of the combination of interventions. For example, the mere presence of dental staff at a nursing home may contribute to unexpected effects on oral care routines, especially if information about oral care is simultaneously disseminated. Therefore, in order to separate the effect of instructions given from the effect of hands-on oral care work, the pilot study was designed with two different intervention groups and a control group. We also wanted to avoid contamination between the study groups due to transfer of information between the nursing staff members. Thus the study groups were clustered in six different nursing homes, two per study group.

So far, most of the studied oral care intervention programs have been evaluated with non-clinical outcomes, such as nursing staff knowledge and attitudes. These outcomes are important, but it is uncertain whether improved knowledge and attitudes lead to improved oral care. Thus, patient-related, oral-health measurements need to be measured and related to subsequent clinical outcomes.

\section{Conclusion}

In conclusion, compared with care as usual, the current trial showed that professional domiciliary oral care resulted in the highest reduction in gingival bleeding, but a significant reduction was also found in the instruction group, in which the highest reduction of dental plaque was found, too. Thus, it seems that both professional oral care and individual instructions need to be combined in a domiciliary oral care intervention programme. Consequently, in an upcoming randomized controlled trial, the effect of the combined intervention will be compared with a control group that receives oral care as usual. In the full-scale trial, we will also follow caries development, general health markers (e.g. pneumonia), oral-health-related quality of life, and nursing-staff knowledge and attitudes. The study material will be collected both at nursing homes and in private homes. The private homes constitute the most challenging context of oral care among care-dependent elderly.

\section{Acknowledgements}

We thank all participants and nursing staff and the dental hygienists that performed the clinical activities, especially the dental hygienist Lena Cederholm, Oral Care, Stockholm, Sweden. Statistical analyses were done by Tommy Johnsson, Institute of Odontology, Sahlgrenska Academy, Gothenburg University, Gothenburg, Sweden 
and language review by Birthe Mousten, Expo-Com ApS, Denmark.

\section{Conflict of Interests}

The authors declare that they have no competing interests.

\section{Funding}

The study was funded by grants from the Stockholm county council.

\section{References}

[1] Meurman, J.H. and Hämäläinen, P. (2006) Oral Health and Morbidity-Implications of Oral Infections on the Elderly. Gerodontology, 23, 3-16. http://dx.doi.org/10.1111/j.1741-2358.2006.00102.x

[2] Marín-Zuluaga, D.J., Sandvik, L., Gil-Montoya, J.A. and Willumsen, T. (2012) Oral Health and Mortality Risk in the Institutionalised Elderly. Medicina Oral, Patología Oral y Cirugía Bucal, 17, 618-623. http://dx.doi.org/10.4317/medoral.17632

[3] Wårdh, I.M. and Wikström, M.B. (2014) Long-Term Effects of Using Oral Care Aides at a Nursing Home for Elderly Dependent Residents-A Pilot Study. Special Care in Dentistry, 34, 64-69. http://dx.doi.org/10.1111/scd.12009

[4] National Guidelines for Dental Care (2010) The National Board of Health and Welfare. (In Swedish)

[5] Lindqvist, L., Seleskog, B., Wårdh, I. and von Bültzingslöwen, I. (2013) Oral Care Perspectives of Professionals in Nursing Homes for the Elderly. International Journal of Dental Hygiene, 11, 298-305. http://dx.doi.org/10.1111/idh.12016

[6] Zuluaga, D.J., Ferreira, J., Montoya, J.A. and Willumsen, T. (2012) Oral Health in Institutionalised Elderly People in Oslo, Norway and Its Relationship with Dependence and Cognitive Impairment. Gerodontology, 29, 420-426. http://dx.doi.org/10.1111/j.1741-2358.2011.00490.x

[7] Wårdh, I., Jonsson, M. and Wikström, M. (2012) Attitudes to and Knowledge about Oral Health Care among Nursing Home Personnel—An Area in Need of Improvement. Gerodontology, 29, 787-792. http://dx.doi.org/10.1111/j.1741-2358.2011.00562.x

[8] Hasson, H. and Arnetz, J.E. (2008) Nursing Staff Competence, Work Strain, Stress and Satisfaction in Elderly Care: A Comparison of Home-Based Care and Nursing Homes. Journal of Clinical Nursing, 17, 468-481.

[9] Belsi, A., Gonzalez-Maffe, J., Jones, K., Wright, D. and Gallagher, J.E. (2013) Care Home Managers’ Views of Dental Services for Older People Living in Nursing and Residential Homes in Inner City London. Community Dental Health, 30, 77-82.

[10] Lundqvist, M., Davidson, T., Ordell, S., Sjöström, O., Zimmerman, M. and Sjögren, P. (2015) Health Economic Analyses of Domiciliary Dental Care and Care at Fixed Clinics for Elderly Nursing Home Residents in Sweden. Community Dental Health, 32, 39-43.

[11] The National Board of Health and Welfare (1998) Särskild Tandvårdsförordning. SFS, Stockholm, 1338. (In Swedish)

[12] Henricsson, V. (1990) Evaluation of a New Device for Measuring Oral Mucosal Surface Friction. Scandinavian Journal of Dental Research, 98, 529-536. http://dx.doi.org/10.1111/j.1600-0722.1990.tb01008.x

[13] Greene, J.C. and Vermillion, J.R. (1964) The Simplified Oral Hygiene Index. The Journal of the American Dental Association, 68, 7-13. http://dx.doi.org/10.14219/jada.archive.1964.0034

[14] Ramfjord, S.P. (1967) The Periodontal Disease Index (PDI). Journal of Periodontology, 38, 602-610. http://dx.doi.org/10.1902/jop.1967.38.6_part2.602

[15] Henriksen, B.M., Ambjørnsen, E. and Axéll, T.E. (1999) Evaluation of a Mucosal-Plaque Index (MPS) Designed to Assess Oral Care in Groups of Elderly. Special Care in Dentistry, 19, 154-157. http://dx.doi.org/10.1111/j.1754-4505.1999.tb01378.x

[16] McArthur, W.P., Bloom, C., Taylor, M., Smith, J., Wheeler, T. and Magnusson, N.I. (1995) Antibody Responses to Suspected Periodontal Pathogens in Elderly Subjects with Periodontal Disease. Journal of Clinical Periodontology, 22, 842-849. http://dx.doi.org/10.1111/j.1600-051X.1995.tb01782.x

[17] Buhlin, K., Gustafsson, A., Håkansson, J. and Klinge, B. (2003) Self-Reported Oral Health, Dental Care Habits and Cardiovascular Disease in an Adult Swedish Population. Oral Health \& Preventive Dentistry, 1, 291-299.

[18] van der Putten, G.J., Mulder, J., de Baat, C., De Visschere, L.M., Vanobbergen, J.N. and Schols, J.M. (2013) Effectiveness of Supervised Implementation of an Oral Health Care Guideline in Care Homes; a Single-Blinded Cluster Randomized Controlled Trial. Clinical Oral Investigations, 17, 1143-1153. http://dx.doi.org/10.1007/s00784-012-0793-2 


\section{Submit or recommend next manuscript to SCIRP and we will provide best service for you:}

Accepting pre-submission inquiries through Email, Facebook, LinkedIn, Twitter, etc.

A wide selection of journals (inclusive of 9 subjects, more than 200 journals)

Providing 24-hour high-quality service

User-friendly online submission system

Fair and swift peer-review system

Efficient typesetting and proofreading procedure

Display of the result of downloads and visits, as well as the number of cited articles

Maximum dissemination of your research work

Submit your manuscript at: http://papersubmission.scirp.org/ 